\title{
Gut bacteria Akkermansia is associated with reduced risk of obesity: evidence from the American Gut Project
}

\author{
Qi Zhou' ${ }^{1}$, Yanfeng Zhang ${ }^{2}$, Xiaoxia Wang ${ }^{3}$, Ruiyue Yang ${ }^{1}$, Xiaoquan Zhu' ${ }^{1}$, Ying Zhang ${ }^{1}$, Chen Chen ${ }^{1}$, \\ Huiping Yuan ${ }^{1}$, Ze Yang ${ }^{1}$ and Liang Sun ${ }^{1,4^{*}}$ (D)
}

\begin{abstract}
Background: Gut bacteria Akkermansia has been shown an anti-obesity protective effect in previous studies and may be used as promising probiotics. However, the above effect may be confounded by common factors, such as sex, age and diets, which should be verified in a generalized population.

Methods: We used datasets from the American Gut Project to strictly reassess the association and further examined the effect of aging on it. A total of 10,534 participants aged 20 to 99 years from the United States and the United Kingdom were included. The relative abundance of Akkermansia was assessed based on $16 \mathrm{~S}$ rRNA sequencing data. Obesity (body mass index, $\mathrm{BMI} \geq 30 \mathrm{~kg} / \mathrm{m}^{2}$ ) risks were compared across Akkermansia quintiles in logistic models with adjustment for common confounders. Restricted cubic splines were used to examine dose response effects between Akkermansia, obesity and age. A sliding-windows-based algorithm was used to investigate the effect of aging on Akkermansia-obesity associations.
\end{abstract}

Results: The median abundance of Akkermansia was $0.08 \%$ (interquartile range: 0.006-0.93\%), and the prevalence of obesity was $11.03 \%$. Nonlinear association was detected between Akkermansia and obesity risk $(P=0.01)$. The odds ratios (95\% confidence interval) for obesity across the increasing Akkermansia quintiles (referencing to the first quintile) were 1.14 (0.94-1.39), $0.94(0.77-1.15), 0.70(0.56-0.85)$ and $0.79(0.64-0.96)$ after adjusting for age and sex ( $P$ for trend $<0.001)$. This association remained unchanged after further controlling for smoking, alcohol drinking, diet, and country. The odds ratios $(95 \% \mathrm{Cl}$ ) of Akkermansia were 0.19 (0.03-0.62) and $0.77(0.64-0.91)$ before and over 40 years, respectively, indicating that the protective effect of Akkermansia against obesity was not stable with aging.

Conclusion: High relative abundance of Akkermansia is associated with low risk of obesity and the association declines with aging.

Keyword: Gut microbiota, $16 \mathrm{~S}$ rRNA, Obesity, Body mass index, Probiotics, Akkermansia

\section{Introduction}

Obesity is associated with or even caused by the dysbiosis of gut microbiota [1]. Some beneficial bacteria, such as gut Akkermansia, may play a decisive role

*Correspondence: sunbmu@foxmail.com

1 The MOH Key Laboratory of Geriatrics, Beijing Hospital, National Center of Gerontology, Beijing 100730, People's Republic of China

Full list of author information is available at the end of the article in reducing the burden of obesity, via modulation of glucose metabolism and low-grade inflammation [2, 3]. Recently, gut bacteria Akkermansia has become a very promising anti-obesity probiotic candidate. The genus and its main species Akkermansia muciniphila inversely correlated with metabolic syndrome $[4,5]$. Treatment with live A. muciniphila (dose $\sim 10^{8}-10^{10}$ per day) was shown to reduce the risk of obesity and original author(s) and the source, provide a link to the Creative Commons licence, and indicate if changes were made. The images or other third party material in this article are included in the article's Creative Commons licence, unless indicated otherwise in a credit line to the material. If material is not included in the article's Creative Commons licence and your intended use is not permitted by statutory regulation or exceeds the permitted use, you will need to obtain permission directly from the copyright holder. To view a copy of this licence, visit http://creativecommons.org/licenses/by/4.0/. The Creative Commons Public Domain Dedication waiver (http://creativeco mmons.org/publicdomain/zero/1.0/) applies to the data made available in this article, unless otherwise stated in a credit line to the data. 
improve insulin resistance, glucose intolerance and steatosis in both mice and humans [6-8]. These findings raise the possibility of new therapeutic strategies for metabolic disorders by targeting the gut microbiota.

However, obesity is an etiologically heterogeneous problem, which is affected by many environmental factors. Such factors can also change the abundance of gut Akkermansia and complicate the Akkermansiaobesity relationship. For example, Akkermansia abundance changes from early childhood to adulthood, varies between geographical locations and differs from health status $[4,5]$. On the other hand, aging has also been shown to be strongly correlated with obesity [9], which may easily confound the relationship with gut Akkermansia. Indeed, some studies have already revealed inconsistent results such as no significant associations between BMI and obesity $[10,11]$. Therefore, before $A$. muciniphila is largely used as a probiotic for treating obesity, three critical questions should be addressed: Firstly, can the potential anti-obesity effects of $A$. muciniphila be generalized to a larger population, especially the elderly? Notably, the effect of $A$. muciniphila on obesity has only been investigated with a small sample size of less than 200 in all prevoius studies. Secondly, how would the common factors such as age, sex, and diet affect obesity and gut microbiota [2, 12-14]? The association changes after adjustment for these confounders remain largely unknown. Thirdly, is there a dose-response effect of Akkermansia on obesity or how does the risk of obesity change when Akkermansia accumulates in the human gut? To answer these questions, a comprehensive population-scale investigation that takes the common environmental confounders into consideration is needed.

The American Gut Project (AGP) was launched in 2012 to characterizes the diversity of the industrialized human gut microbiome [15]. This project was carried out at an unprecedented scale which included more than 10,000 human participants and yielded over 467 million (48,599 unique) $16 \mathrm{~S}$ rRNA V4 gene sequencing data. Most importantly, it covers data on microbial compositions in different common factors, and the temporal and spatial stability of gut microbiota, which thereby providing a valuable opportunity to investigate the effect of Akkermansia on obesity in a large population under complex environmental conditions.

In this study, we performed a cross-sectional study based on the AGP database of 10,534 subjects. The association between Akkermansia and the risk of obesity was estimated with adjusting for age, sex, smoking, alcohol drinking, diet and country. We also investigated the effect of aging on the Akkermansia-obesity association.

\section{Materials and methods}

\section{Study population}

This study is a cross-sectional analysis of baseline data from the AGP study, which were initially to explore the relationships between gut microbiota and human health. The health status was self-reported and the microbiota analysis was performed by high-throughput sequencing data of $16 \mathrm{~S}$ rRNA genes (V4 fragments). The data for the participants from the United Kingdom and the United States were used in this study. Subjects with missing body mass index (BMI); missing sex; BMI $>50 \mathrm{~kg} / \mathrm{m}^{2}$ or $\mathrm{BMI}<10 \mathrm{~kg} / \mathrm{m}^{2}$; number of qualified sequencing reads $<5000$; age $<20$ years old were excluded. Finally, a total of 10,534 individuals (5688 females and 4846 males) aged 20 to 99 years were obtained for the present analyses.

\section{Assessment of obesity}

Body mass index (BMI) was calculated as weight in kilograms divided by the square of height in meters. Obesity was identified and classified by BMI according to WHO definitions [16]: normal weight as a reference $\left(\mathrm{BMI}<25 \mathrm{~kg} / \mathrm{m}^{2}\right.$ ), overweight (BMI is $\geq 25$ but $<30 \mathrm{~kg} /$ $\left.\mathrm{m}^{2}\right)$, and obesity $\left(B M I \geq 30 \mathrm{~kg} / \mathrm{m}^{2}\right)$.

\section{Assessment of Akkermansia abundance and covariates}

The relative abundance of Akkermansia was obtained by processing the $16 \mathrm{~S}$ rRNA sequencing reads using Vsearch-2.1.4.2 software with default arguments [17]. Briefly, raw reads were qualified into clean reads with a max error rate of 0.01 , clustered into Operational Taxonomic Units (OTUs, similarity threshold 97\%), and then contrasted into OTUs tables. Representative OTUs were classified into taxon (sintax_cutoff, 0.8 ) by using the Silva_16s_v123 database [18]. Relative abundances of Akkermansia were assessed by merging the OTUs and taxonomy tables. Samples with $<5000$ clean reads were discarded.

Age, sex, smoking frequency, alcohol drinking frequency, diet type, and country (the United States, the United Kingdom.) data were obtained from the baseline questionnaire. Smoking status was grouped into never, occasionally (a few times/month), and regularly ( $\geq 3-5$ times/week). Drinking frequency was classified into never, occasionally ( $\leq 1-2$ times/week), and regularly $(\geq 3-5$ times/week). The diet types were classified into vegan and nonvegan.

\section{Statistical analyses}

We reported baseline characteristics of this study across Akkermansia quintiles. Categorical variables were summarized using absolute numbers and percentages, whereas continuous variables were summarized using the median and interquartile range, or using the mean 
and standard deviation. Multivariate linear regression or logistic regression were performed for the comparison of common cofounders across Akkermansia quintiles.

The correlation between BMI, Akkermansia relative abundance and age was tested by Pearson correlation test. Wilcoxon rank-sum test was used to analyze the Akkermansia differences between subjects of normal weight, overweight and obesity. Multivariate linear regression or logistic regression was applied for the comparison of common cofounders across the three BMI groups.

Logistic regression models were used to test odds ratios (ORs) and confidence intervals (CIs) of overweight and obesity for each Akkermansia quintile compared with the lowest one. Models were first adjusted for age (continuous) and sex and were further adjusted for smoking frequency (ordered category variables), alcohol drinking frequency (ordered category variables), diet type (vegan, nonvegan), and country (United Kingdom., United States.). We performed tests for linear trend by entering the median value of each category of Akkermansia relative abundance as a continuous variable in the models. The dose-response relationship was estimated by applying a restricted cubic spline model with 2 knots at the 5th $(0.08 \%)$ and 50 th $(3.7 \%)$ percentiles of the Akkermansia relative abundance.
Stratified analyses were performed according to age ( $<65$ years, $\geq 65$ years), sex, smoking frequency, alcohol drinking frequency, diet type, and country. Likelihood ratio tests were conducted to examine interactions between Akkermansia and each of the confounders.

We used a modified SWAN (sliding window analysis) algorithm [19] to identify and quantify nonlinear changes of the Akkermansia-obesity association during aging. This algorithm analyzes odds ratios in fully adjusted logistic models within a window of 10 years (e.g., 20-30 years, $30-40$ years and so on), while sliding the window in increments of 1 year from young to old. Windows of 5 years and 20 years were also used to compare the robustness of SWAN algorism. Linear regression model was used to test whether the odds ratios were significantly changed with age and group differences of ORs were compared by Mann-Whitney U-tests. For all analyses and plots, we used $\mathrm{R} \times 64$ 3.6.1 and the related packages. All $P$ values were 2 -sided.

\section{Results}

\section{Characteristics of study participants}

The characteristics of study participants are presented in Table 1 . The average age was approximately $53 \pm 15$ years, and approximately $54.0 \%$ was female. $92.8 \%$ of the participants were nonsmokers, $17.5 \%$ did not drink alcohol,

Table 1 Characteristics of participants according to quintile of Akkermansia abundance $(n=10,534)$

\begin{tabular}{|c|c|c|c|c|c|c|}
\hline \multirow[t]{2}{*}{ Characteristics $^{\text {a }}$} & Q1 & Q2 & Q3 & Q4 & & $P$ for trend $^{b}$ \\
\hline & $0-0.003$ & $0.003-0.022$ & $0.022-0.023$ & $0.024-1.40$ & $1.4-49.8$ & \\
\hline $\mathrm{n}$ & 2107 & 2107 & 2106 & 2107 & 2107 & \\
\hline Age (years) & $50.4 \pm 15$ & $50.5 \pm 14.8$ & $52.5 \pm 14.5$ & $53.4 \pm 14.7$ & $56.2 \pm 14.3$ & $<0.001$ \\
\hline Sex, male, n (\%) & $988(46.9)$ & 1079 (51.2) & 1024 (48.6) & $912(43.3)$ & $843(40.0)$ & $<0.001$ \\
\hline BMI $\left(\mathrm{kg} / \mathrm{m}^{2}\right)$ & $24.5 \pm 5.2$ & $24.9 \pm 4.9$ & $24.7 \pm 4.8$ & $24.2 \pm 4.5$ & $24.4 \pm 4.7$ & $<0.001$ \\
\hline Normal, n (\%) & 1326 (62.9) & $1274(60.5)$ & $1306(62.0)$ & 1358 (64.5) & 1302 (61.8) & \\
\hline Overweight, n (\%) & $535(25.4)$ & $563(26.7)$ & $553(26.3)$ & $562(26.7)$ & $593(28.1)$ & \\
\hline Obesity, n (\%) & $246(11.7)$ & $270(12.8)$ & $247(11.7)$ & $187(8.9)$ & $212(10.1)$ & \\
\hline Smoking, $\mathrm{n}(\%)^{\mathrm{c}}$ & & & & & & 0.01 \\
\hline Never & 1915(90.9) & $1919(91.1)$ & $1960(93.1)$ & $1994(94.6)$ & 1997 (94.8) & \\
\hline Occasionally & $110(5.2)$ & $120(5.7)$ & $102(4.8)$ & $69(3.3)$ & $66(3.1)$ & \\
\hline Regularly & $68(3.2)$ & $49(2.3)$ & $37(1.8)$ & $32(1.5)$ & $30(1.4)$ & \\
\hline Alcohol drinking, $n(\%)^{d}$ & & & & & & 0.23 \\
\hline Never & $416(19.7)$ & $390(18.5)$ & $330(15.7)$ & $327(15.5)$ & $385(18.2)$ & \\
\hline Occasionally & $1004(47.7)$ & $1032(49.0)$ & 1027 (48.8) & $1032(49.0)$ & 981 (46.6) & \\
\hline Regularly & $668(31.7)$ & $669(31.8)$ & $740(35.1)$ & 729 (34.6) & $727(34.5)$ & \\
\hline Diet, vegan, n (\%) ${ }^{e}$ & $134(6.4)$ & $182(8.6)$ & $120(5.7)$ & $138(6.5)$ & $114(0.54)$ & 0.02 \\
\hline Country, U.K., n (\%) & $640(30.3)$ & $592(28.1)$ & $657(31.2)$ & $665(31.6)$ & $668(31.7)$ & 0.40 \\
\hline
\end{tabular}

\footnotetext{
Data are means \pm SD or $n(\%)$

b $P$-value was calculated after adjustment for age, sex, except for itself

$c, d$, e Data are missing for $66,77,124$ participants, respectively

$f$ Range of the relative abundances of Akkermansia (\%)
} 
$6.5 \%$ of the sample was vegetarian, and $30.6 \%$ of the sample were from the United Kingdom. A total of $26.6 \%$ of the participants were overweight, and $11.0 \%$ of the participants had obesity. The median relative abundance of Akkermansia was $0.08 \%$ (interquartile range: $0.006-$ $0.93 \%$ ), and no Akkermansia was detected in $17.6 \%$ subjects. Participants who had higher Akkermansia relative abundances were more likely to be younger, female, smokeless and nonvegetarians. There was a strong correlation between Akkermansia and age: Akkermansia abundance was significantly increased with age in an adjusted linear regression model $(P$ for trend $<0.001)$. However, geographic location and alcohol drinking frequency have no significant impact on the abundance of gut Akkermansia (both $P>0.05$ ). The age, sex, smoking frequency and vegan percentage were significantly different between the three BMI groups (Additional file 1). For instance, the
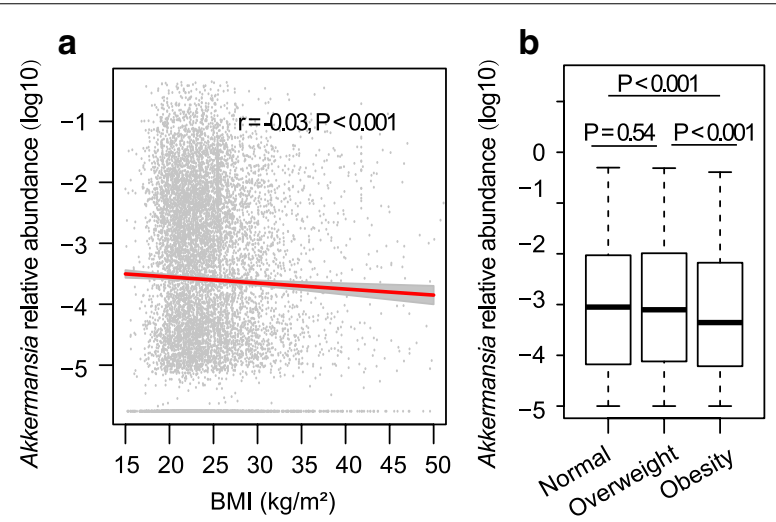

Fig. 1 The association between BMl and relative abundance of Akkermansia. a Pearson correlation between BMI (as a continuous variable) and Akkermansia abundance. b The Wilcoxon rank-sum test was used to analyze the Akkermansia differences between three BMI categories, namely Normal, Overweight, and Obesity. The $\log _{10}$-transformed Akkemancia relative abundance was used in plots average age of subjects with obesity was 55.5 compared to 50.8 for those with a normal BMI.

\section{Akkermansia and risk of obesity}

The BMI and the relative abundance of Akkermansia were inversely correlated across the entire study population ( $r=-0.03, P<0.001$ ) (Fig. 1a), indicating a weak but significant protective effect on gut Akkermansia against obesity. Participants with obesity had reduced Akkermansia compared to participants with a normal BMI (median relative abundance: $0.088 \%$ in normal weight versus $0.043 \%$ in obesity, $P<0.001$ ) (Fig. 1b). However, the differences between overweight group and normal weight group were not statistically significant (median relative abundance: $0.088 \%$ in normal weight versus $0.077 \%$ in overweight, $P=0.54$ ) (Fig. $1 \mathrm{~b}$ ).

Higher abundance of Akkermansia was associated with lower risk of obesity, which was independent of some common confounders (Table 2). After adjusting for age and sex, the ORs $(95 \% \mathrm{CI})$ for obesity from the lowest to the highest Akkermansia relative abundance quintiles were 1.14 (0.94-1.39), 0.94 (0.77-1.15), 0.70 (0.56-0.85) and 0.79 (0.64-0.96) (Model 3). The Akkermansia-obesity association was not substantially changed $(P$ for trend $<0.001)$ after further adjusting for smoking frequency, alcohol drinking frequency, diet type, and country (Model 4), suggesting that the protective effect of higher Akkermansia abundance against obesity risks is independent of other confounding factors. Furthermore, analysis of dose-response effects revealed a consistent trend toward lower obesity risk for higher Akkermansia abundance, and the Akkermansia-obesity association was significantly nonlinear $(P=0.01)$ (Fig. 2). However, protective effect was not seen in the overweight group ( $P$ for trend $=0.13$ and 0.15 in Model 1 and Model 2, respectively). The ORs (95\% CI) for overweight from the lowest to the highest quantile of Akkermansia relative

Table 2 Odds ratio (95\% confidence interval) of overweight/obesity according to quintiles of Akkermansia relative abundance

\begin{tabular}{|c|c|c|c|c|c|c|}
\hline & $\mathrm{Q} 1^{\mathrm{c}}$ & Q2 & Q3 & Q4 & Q5 & $\mathrm{P}$ trend \\
\hline & $0-0.003$ & $0.003-0.022$ & $0.022-0.023$ & $0.024-1.40$ & $1.4-49.8$ & \\
\hline Overweight / Normal (n) & $535 / 1326$ & $563 / 1274$ & $553 / 1306$ & $562 / 1358$ & $593 / 1302$ & \\
\hline Model $1^{\mathrm{a}}$ & 1 & $1.05(0.91-1.21)$ & $1.00(0.87-1.16)$ & $0.98(0.85-1.13)$ & $1.04(0.90-1.21)$ & 0.13 \\
\hline Model $2^{b}$ & 1 & $1.06(0.91-1.23)$ & $0.99(0.86-1.16)$ & $0.98(0.84-1.13)$ & $1.04(0.89-1.20)$ & 0.15 \\
\hline Obesity / Normal (n) & $246 / 1326$ & $270 / 1274$ & 247/1306 & $187 / 1358$ & $212 / 1302$ & \\
\hline Model $3^{\mathrm{a}}$ & 1 & $1.14(0.94-1.39)$ & $0.94(0.77-1.15)$ & $0.70(0.56-0.85)$ & $0.79(0.64-0.96)$ & $<0.001$ \\
\hline Model $4^{\mathrm{b}}$ & 1 & $1.16(0.97-1.42)$ & $0.96(0.79-1.17)$ & $0.71(0.57-0.87)$ & $0.78(0.63-0.95)$ & $<0.001$ \\
\hline
\end{tabular}

\footnotetext{
a Model 1 and Model 3 were adjusted for age and sex

b Model 2 and Model 4 were additionally adjusted for diet type, smoking and alcohol drinking and country

c Range of the relative abundances of Akkermansia (\%)
} 


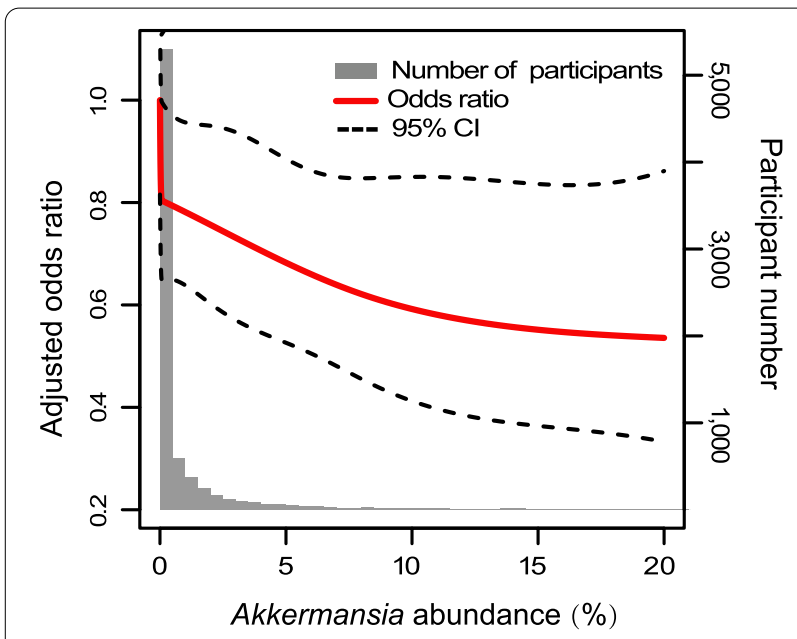

Fig. 2 Odds ratio of obesity by relative abundance of Akkermansia. Lines represent odds ratios and $95 \% \mathrm{Cl}$ based on restricted cubic splines for relative abundance of Akkermansia with knots at the 50th and 90th percentiles. Odds ratios were estimated using a logistic regression model after adjustment for age, sex, diet type, smoking and alcohol drinking and country; $P$ for non-linearity $=0.01$. Bars represent the numbers of participants according to 100-equally-sized bins of Akkermansia abundance

abundance were 1.05 (0.91-1.21), 1.00 (0.87-1.16), 0.98 $(0.85-1.13)$, and $1.04(0.90-1.21)$ in Model 1 with adjustment for age and sex. A similar result was found in the fully adjusted Model 2.

To confirm the above observed Akkermansia-obesity association, stratified analysis was performed (Fig. 3). The association can still be observed when common factors were stratified. However, it was slightly stronger in the elderly, smokers, alcohol drinkers, and non-vegetarians, but no significant interactions were detected with these variables $(P$ for interaction $>0.1$ ).

We finally calculated the risk of obesity in a fully adjusted model with Akkermansia as a continuous variable. The results showed that $10 \%$ higher Akkermansia would reduce the risk for obesity approximately $26 \%$, on average (OR: 0.74, CI: 0.61-0.84) (Fig. 3). Risks of overweight for a $10 \%$ increase of Akkermansia was also estimated. In line with the above results, the association between Akkermansia and overweight was not statistically significant $(P=0.1)$.

\section{Decreased anti-obesity effect of Akkermansia with aging}

Aging has been known as a strong confounder of the Akkermansia-obesity association. In the present study, $\mathrm{BMI}$ and Akkermansia were both positively correlated with age (BMI: $\mathrm{r}=0.13, P<0.001$; Akkermansia: $\mathrm{r}=0.07$, $P<0.001$ ) (Additional file 2a, b). Besides, a significant nonlinear association was also detected between age and obesity risks $(P$ for nonlinearity $<0.001)$ (Additional file 2c).

To further understand the aging effects, we compared the alterations in obesity risks after a 10\% increase in abundance of Akkermansia across different age groups using SWAN algorithm. The results showed that aging weakened the protective effect of Akkermansia against obesity (Fig. 4). The OR value significantly increased with age and reached a plateau at approximately 40 years old in models controlled for age and sex (before 40 years old, $P<0.0001$; after 40 years old, $P=0.709$ ) (Fig. 4 a) or models that additionally controlled for the other confounders (before 40 years old, $P<0.0001$; after 40 years old, $P=0.08)$ (Fig. 4b).

The protective effect of Akkermansia before 40 years was significantly smaller than after 40 years (Table 3 , Additional file 3): In fully adjusted models (Model 2), the ORs $(95 \% \mathrm{CI})$ in subjects of $<40$ years and $\geq 40$ years were $0.19(0.03-0.62)$ and $0.77(0.64-0.91)$, respectively, and ORs (95\% CI) in Model 1 were 0.20 (0.04-0.65) and $0.78(0.64-0.92)$, respectively, in the two age groups. To confirm the robustness of the SWAN algorithm, we set the width of the slide window to 5 years or 20 years, and consistent findings were observed between different models (Additional file 4).

\section{Discussion}

To our knowledge, this study is the first large populationbased study showing that decreased gut Akkermansia was associated with an increased risk of obesity, which is independent of age, sex, smoking, alcohol drinking, diet and country. We also found that the association changed with aging.

Most previous studies found that the depletion of Akkermansia-like spp. was strongly associated with obesity and metabolic disorders, but some others showed that the level of $A$. muciniphila did not correlate with $B M I$ value $[10,11]$. Our large-scale population estimates showed that increased BMI and risk of obesity were correlated with decreased relative abundance of Akkermansia. Such discrepancy may come from some confounders, especially when the sample size was small. Many common factors such as age, sex, geography and ethnic origins shaped the gut microbiota [13, 20,21], and were closely related to obesity. Therefore, it is necessary to assess the independent effect of Akkermansia on obesity in large populations. We found that the association between the risk of overweight and Akkermansia relative abundance was not statistically significant in all of the models. No relevant results were mentioned in animal or human studies. The "Enterotype" of normal weight subjects is more similar to that of overweight subjects than 


\begin{tabular}{|c|c|c|c|c|c|}
\hline \multirow{2}{*}{$\begin{array}{l}\text { Participant } \\
\text { subgroups }\end{array}$} & \multicolumn{2}{|c|}{ Akkermansia relative abundance ${ }^{a}$} & \multirow{2}{*}{$\begin{array}{l}\text { Odds ratio } \\
(95 \% \mathrm{Cl})^{\mathrm{b}}\end{array}$} & & \multirow{2}{*}{$P$ for interaction } \\
\hline & $\begin{array}{l}\text { Participants of } \\
\text { normal weight }\end{array}$ & $\begin{array}{l}\text { Participants } \\
\text { with obesity }\end{array}$ & & & \\
\hline Age & & & & & 0.64 \\
\hline$<65$ & $0.07(0.005-0.81)$ & $0.03(0.005-0.64)$ & $0.84(0.68-1.01)$ & $\mapsto-$ & \\
\hline$\geq 65$ & $0.18(0.009-1.42)$ & $0.06(0.004-0.72)$ & $0.61(0.40-0.86)$ & $\longmapsto$ & \\
\hline Sex & & & & & 0.82 \\
\hline Male & $0.13(0.007-1.20)$ & $0.04(0.005-0.84)$ & $0.73(0.57-0.89)$ & $\mapsto$ & \\
\hline Female & $0.09(0.006-0.93)$ & $0.04(0.005-0.63)$ & $0.73(0.61-0.86)$ & $\mapsto$ & \\
\hline Smoking & & & & & 0.65 \\
\hline Never & $0.01(0.006-0.97)$ & $0.05(0.005-0.71)$ & $0.74(0.61-0.88)$ & $\mapsto-1$ & \\
\hline Occasionally & $0.01(0.000-0.31)$ & $0.03(0.000-0.33)$ & $0.80(0.32-1.59)$ & & \\
\hline Regularly & $0.03(0.004-0.48)$ & $0.009(0.003-0.14)$ & $0.60(0.30-0.93)$ & - & \\
\hline Alcohol drinking & & & & & 0.16 \\
\hline Never & $0.06(0.004-0.85)$ & $0.06(0.006-1.28)$ & $0.90(0.64-1.20)$ & $\longmapsto$ & $\longrightarrow$ \\
\hline Occasionally & $0.09(0.006-1.02)$ & $0.04(0.004-0.55)$ & $0.67(0.51-0.85)$ & $\longmapsto$ & \\
\hline Regularly & $0.10(0.007-0.85)$ & $0.04(0.006-0.53)$ & $0.65(0.39-0.98)$ & $\longmapsto$ & \\
\hline Diet & & & & & 0.99 \\
\hline Vegan & $0.04(0.005-0.61)$ & $0.02(0.0005-4.1)$ & $0.81(0.25-1.55)$ & & -1 \\
\hline Nonvegan & $0.09(0.006-0.96)$ & $0.04(0.005-0.67)$ & $0.74(0.60-0.87)$ & $\mapsto-1$ & \\
\hline Country & & & & & 0.91 \\
\hline UK & $0.13(0.007-1.08)$ & $0.05(0.005-0.69)$ & $0.70(0.49-0.94)$ & $\longmapsto$ & \\
\hline USA & $0.07(0.005-0.86)$ & $0.04(0.005-0.66)$ & $0.75(0.59-0.91)$ & $\longmapsto 1$ & \\
\hline \multirow[t]{3}{*}{ Overall } & $0.09(.0 .006-0.93)$ & $0.04(0.005-0.66)$ & $0.74(0.61-0.87)$ & $1=-1$ & \\
\hline & & & & \multirow{2}{*}{\multicolumn{2}{|c|}{$\begin{array}{ccc}0.5 & 1 & 1.5 \\
\text { Odds ratio } & (95 \% \mathrm{Cl})\end{array}$}} \\
\hline & & & & & \\
\hline
\end{tabular}

Fig. 3 Stratified analyses of the associations between fecal relative abundance (\%) of Akkermansia and obesity. ${ }^{2}$ Range of the relative abundances of Akkermansia was revealed by median (interquartile range). ${ }^{b}$ Adjusted for age, sex, diet type, smoking and alcohol drinking and country, stratifying factors excepted

that of subjects with obesity according to Akkermansia distribution.

We adjusted for age and sex in all models because these factors influence obesity prevalence and the gut microbiota $[11,13]$. Age shifted the core gut microbiota in both composition and diversity and may confuse relationships between microbiota and diseases. One previous report showed that the aging-associated microbiome masked the microbial signatures of colorectal cancer because of the interactions between cancer-microbiome and aging [22]. The A. muciniphila abundance was significantly increased from early life to adult subjects and then reduced in elderly subjects [23]. We found that the protective effect of Akkermansia decreased with aging: it kept decreasing from young adults to middleaged (40 years old). This result indicates that aging may weaken the protective effect which deserves attention in further experiments or clinical trials. The risks of obesity between genders were not statistically different in our study.

We also found a nonlinear association between obesity risk and Akkermansia abundance in two models where the relative abundance of Akkermansia was treated as continuous or categorical variables, respectively. This 

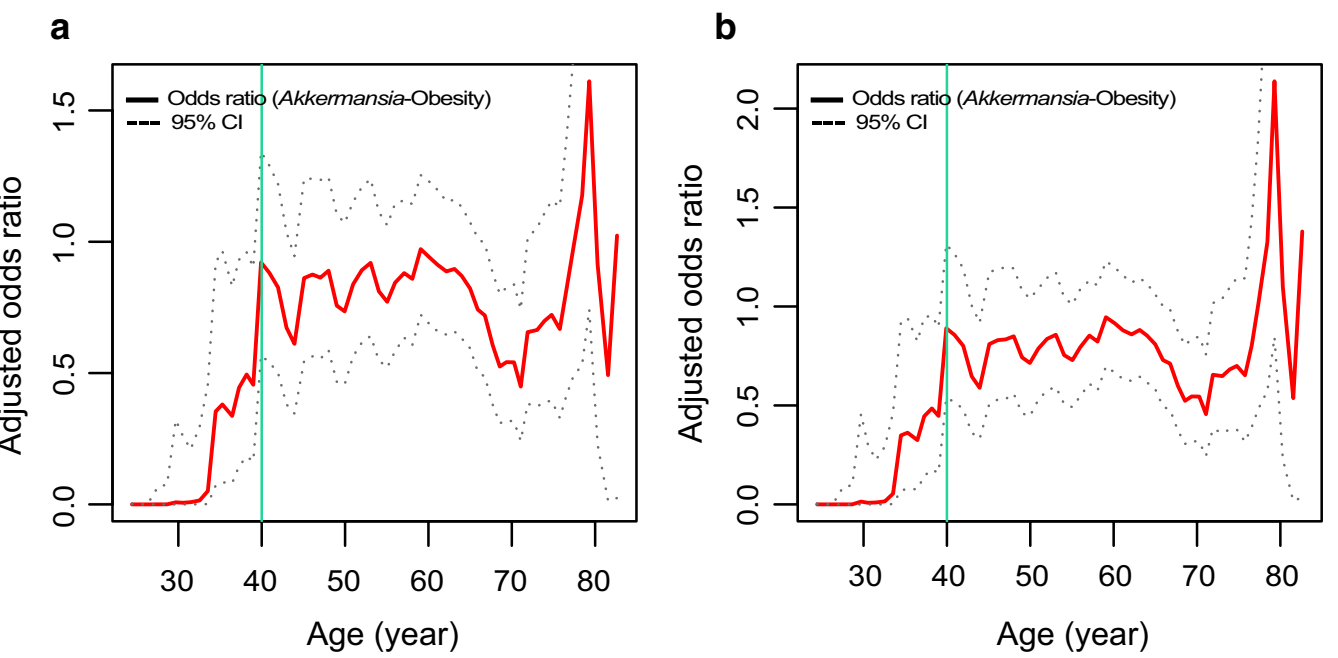

Fig. 4 The effect of aging on Akkermansia-obesity associations. a The changing ORs (between Akkermansia and obesity) with age in sliding window analysis. The OR represented obesity risks of elevating of per 10\% Akkermansia abundance in age- and sex-adjusted (a), or in fully adjusted logistic regression models (b). Lines represent odds ratios and $95 \% \mathrm{Cl}$

Table 3 Odds ratio ${ }^{\#}$ (95\% confidence interval) of obesity before and after $\mathbf{4 0}$ years old

\begin{tabular}{lll}
\hline & \multicolumn{2}{l}{ Age groups (year) } \\
\cline { 2 - 3 } & $\mathbf{2 0 - 3 9}$ & $\mathbf{4 0 - 9 9}$ \\
\hline BMl & $23.3 \pm 4.2$ & $24.9 \pm 5.0$ \\
Normal, n, (\%) & $1752(74.7)$ & $1752(58.8)$ \\
Overweight, n, (\%) & $445(19.0)$ & $445(28.8)$ \\
Obesity, n, (\%) & $149(6.4)$ & $149(12.4)$ \\
Model 1 ${ }^{\text {a }}$, OR (95\% Cl) & $0.20(0.04-0.65)^{* * *}$ & $0.78(0.64-0.92)$ \\
Model 2 ${ }^{\text {b }}$, OR (95\% Cl) & $0.19(0.03-0.62)^{* * *}$ & $0.77(0.64-0.91)$ \\
\hline
\end{tabular}

a Model 1 was adjusted for sex

b Model 2 was additionally adjusted for diet type, smoking and alcohol drinking and country

\# The Obesity risk of elevating $10 \%$ abundance of Akkermansia

*** OR differences between age groups were significant with $P$ values of $<0.0001$ by Mann-Whitney $U$-test

result has not been mentioned before. The Akkermansia dose-response effects in metabolic dysfunction have been noted in animal models: the live A. muciniphila must be used at doses $>4.0 \times 10^{7} \mathrm{CFU} /$ day to exert their beneficial effect [24]. Other interventional studies, in humans and mice, used $10^{8}-10^{10} \mathrm{CFU} /$ day to improve metabolic diseases $[7,8]$. There are no published clinical trials of $A$. muciniphila in humans, which results in a lack of evidence on what dose of the bacteria is beneficial and safe in humans. Administration of A. muciniphila at $10^{8} \mathrm{CFU} /$ day has been proved to be safe for humans [7], and there were no adverse events that occurred in people whose relative level of $A$. muciniphila reached $60 \%$
[25]. We observed a significant reduction of obesity risk when the relative abundance of Akkermansia reached the fourth quintile $(0.24 \%)$.

One previous study reported that $A$. muciniphila was detected in $75 \%$ of European populations [23], while we found $\sim 82 \%$ of the populations had Akkermansia. The frequency of $A$. muciniphila was lower in Southern Chinese $(\sim 52 \%)$ [26] than the European population. However, the Akkermansia was similar between people in the United Kingdom $(\sim 82 \%)$ and the United States $(\sim 83 \%)$ in the present study. These results could be due to the differences between the Western and Eastern diets. The median relative abundance of gut Akkermansia in our population was $0.08 \%$ (interquartile range: $0.006-0.9 \%$ ), while in the other studies, the relative abundance of $A$. muciniphila was significantly different, accounting for $<0.01-5 \%[5,6,14]$. The explanation for the wide range of Akkermansia and Akkermansia-like bacteria is not known. It may result from different methods for bacterial identification (e.g., in situ hybridization, qPCR sequencing, whole-genome shotgun sequencing and 16S rRNA sequencing). The high-throughput sequencing, which detects more unculturable bacteria, may result in a lower relative abundance of Akkermansia than the other methods.

As with any observational study, there are several limitations in the present study: (i) Clinical records on some confounders such as cancer, hypertension, and treatment with metformin were not available. For example, metformin administration increased Akkermansia abundance in obese mice and type 2 diabetic subjects [27, 28]. (ii) Our findings were based on subjects who consumed 
the Western diet, which may reduce the generalizability of this result. Although we adjusted the diet type (vegan or nonvegan), validation in subjects consuming Eastern diet is recommended. Besides, due to the nature of selfreported data, the classification of some factors, such as smoking frequency may induce errors into the analysis. (iii) The Akkermansia-obesity association (genus level) does not represent the association between $A$. muciniphila and obesity (species level). A. muciniphila is the major species in the genus of Akkermansia, and both of them exhibit strong associations with obesity. We focused on Akkermansia, because the species level may not be accurately annotated by sequencing the 16S rRNA V4 fragment. The genus-level rather than the species-level is frequently preferred in high-throughput $16 \mathrm{~S}$ rRNA sequencing studies.

Our study has several key strengths. First, the sample size ( $>10,000$ participants) is larger than any of the previous studies, to our knowledge. Second, the adjustment of several important confounders especially age in models provided a clearer understanding of the Akkermansiaobesity association than previous studies. Third, it is the first analysis that systematically reveals the undulating changes in Akkermansia-obesity relationships across the lifespan.

\section{Conclusions}

Based on a large dataset from AGP and by accounting for common confounders such as sex, age and diet, we provided evidence that a higher relative abundance of Akkermansia may be associated with a low risk of obesity and this association seems to decline with aging.

\section{Supplementary information}

Supplementary information accompanies this paper at https://doi. org/10.1186/s12986-020-00516-1.

Additional file 1. Characteristics of participants among BMI groups.

Additional file 2. The correlations of age with Akkermansia, BMI and obesity risk.

Additional file 3. The differences of ORs before and after 40 years old in age-and sex-adjusted and fully adjusted models.

Additional file 4. The effect of aging on Akkermansia-obesity associations using SWAN algorithm with 5-years-, 10-years- and 20-years-sliding windows.

\section{Abbreviations}

BMI: Body mass index; OTUs: Operational Taxonomic Units; 95\% Cl: 95\% Confidence interval; OR: Odds ratio; SD: Standard deviation; SWAN: Sliding window analysis; AGP: American Gut Project.

\section{Acknowledgements}

We are very grateful to Dr. Yong Jia from Murdoch Universtiy and Dr. Jun Xiong from Institute of Biophysics, Chinese Academy of Sciences for their continuous support during the preparation and revision of the manuscript. We thank all the researchers and volunteers in the AGP for providing such an excellent dataset that could be shared by scientists all over the world.

\section{Authors' contributions}

LS and QZ conceived the study; QZ and YFZ wrote the manuscript; YZ and CC performed the statistical analyses; HPY, XXW, ZY, XQZ, and JML contributed to the critical review of the manuscript; $Q Z$ and RYY participated in the acquisition of the data; QZ and LS had full access to all the data in the study and takes responsibility for the integrity of the data and the accuracy of the data analysis, and all authors read and approved the final manuscript.

\section{Funding}

This research was funded by the National Scientific Foundation of P. R. China (91849132, 81571385, 81870552 and 81400790), the Doctoral Scientific Research Foundation of Beijing Hospital (BJ-2018-024), Non-profit Central Research Institute Fund of Chinese Academy of Medical Sciences (2018RC330003), CAMS Innovation Fund for Medical Sciences (2018-12M1-002), the Beijing Hospital Nova Project (BJ-2018-139), National Key R\&D Program of China (2018YFC2000400), and Priority Union Foundation of Yunnan Provincial Science and Technology Department and Kunming Medical University (202001AY070001-011).

\section{Availability of data and materials}

The data of this study were downloaded from EBI under project PRJEB1 1419

\section{Ethics approval and consent to participate}

The human data were obtained from the publish database of AGP [15] which was performed in accordance with the University of Colorado Boulder's Institutional Review Board Protocol Number 12-0582 and the University of California San Diego's Human Research Protection Program Protocol Number 141853.

\section{Consent for publication}

Not applicable.

\section{Competing interests}

The authors declare that they have no competing interests.

\section{Author details}

${ }^{1}$ The MOH Key Laboratory of Geriatrics, Beijing Hospital, National Center of Gerontology, Beijing 100730, People's Republic of China. ${ }^{2}$ University of Chinese Academy of Sciences, Beijing 100049, People's Republic of China. ${ }^{3}$ Department of Endocrinology, Beijing Hospital, National Center of Gerontology, Beijing 100730, People's Republic of China. ${ }^{4}$ NHC Key Laboratory of Drug Addiction Medicine, Kunming Medical University, Kunming 650032, People's Republic of China.

Received: 30 April 2020 Accepted: 16 October 2020

Published online: 22 October 2020

\section{References}

1. Zhao L. The gut microbiota and obesity: from correlation to causality. Nat Rev Microbiol. 2013;11(9):639.

2. Gérard and Philippe. Gut microbiota and obesity. Cell. Mol. Life Sci. 2016; 73(1):147-62.

3. Turnbaugh PJ, Ley RE, Mahowald MA, Magrini V, Mardis ER, Gordon JI. An obesity-associated gut microbiome with increased capacity for energy harvest. Nature. 2006;444(7122):1027-131.

4. Cani PD, de Vos WM. Next-generation beneficial microbes: the case of Akkermansia muciniphila. Front Microbiol. 2017;8:1765.

5. Zhang T, Li Q, Cheng L, Buch $H$, Zhang F. Akkermansia muciniphila is a promising probiotic. Microb Biotechnol. 2019;12:1109-25.

6. Everard A, Belzer C, Geurts L, Ouwerkerk JP, Druart C, Bindels LB, Guiot Y, Derrien M, Muccioli GG, Delzenne NM. Cross-talk between Akkermansia muciniphila and intestinal epithelium controls diet-induced obesity. Proc Natl Acad Sci USA. 2013;110:9066-71.

7. Depommier C, Everard A, Druart C, Plovier H, Hul MV, Vieira-Silva S. Falony G, Raes J, Maiter D, Delzenne NM et al. Supplementation with 
Akkermansia muciniphila in overweight and obese human volunteers: a proof-of-concept exploratory study. Nat. Med. 2019; 25(7):1096.

8. Plovier H, Everard A, Druart C, Depommier C, Van Hul M, Geurts L, Chilloux J, Ottman N, Duparc T, Lichtenstein L, et al. A purified membrane protein from Akkermansia muciniphila or the pasteurized bacterium improves metabolism in obese and diabetic mice. Nat Med. 2017;23(1):107-13.

9. Jura M, Kozak LP. Obesity and related consequences to ageing. Age. 2016;38(1):23

10. Santacruz A, Collado MC, García-Valdés L, Segura MT, Sanz Y. Gut microbiota composition is associated with body weight, weight gain and biochemical parameters in pregnant women. Br J Nutr. 2010;104(1):83-92.

11. Escobar JS, Klotz B, Valdes BE, Agudelo GM. The gut microbiota of Colombians differs from that of Americans, Europeans and Asians. BMC Microbiol. 2014;14(1):311.

12. Derrien M, Belzer C, de Vos WM. Akkermansia muciniphila and its role in regulating host functions. Microbiol Pathog. 2017;9:171-81.

13. de la Cuesta-Zuluaga J, Kelley ST, Chen Y, Escobar JS, Mueller NT, Ley RE, McDonald D, Huang S, Swafford AD, Knight R, Thackray VG. Age- and sex-dependent patterns of gut microbial diversity in human adults. mSystems 2019: 4(4):e00261-19.

14. Geerlings S, Kostopoulos I, De Vos W, Belzer C. Akkermansia muciniphila in the human gastrointestinal tract: when, where, and how? Microorganisms. 2018;6(3):75

15. McDonald D, Hyde E, Debelius J.W et al. American gut: an open platform for citizen science microbiome research. mSystems 2018; 3(3):e00031-00018.

16. World Health Organization: Global Database on Body Mass Index (BMI). https://www.who.int/nu-trition/databases/bmi/en/ (30 March 2020, date last accessed).

17. Rognes T, Flouri T, Nichols B, Quince C, Mahé F. VSEARCH: a versatile open source tool for metagenomics. PeerJ. 2016;4:e2584-e2584.

18. Quast C, Pruesse E, Yilmaz P, Gerken J, Schweer T, Yarza P, Peplies J, Glockner FO. The SILVA ribosomal RNA gene database project: improved data processing and web-based tools. Nucleic Acids Res. 2013;4(10):D590-596.

19. Lehallier $B$, Gate $D$, Schaum N, et al. Undulating changes in human plasma proteome profiles across the lifespan. Nat Med. 2019;25:1843-50.
20. Biagi E, Candela M, Turroni S, Garagnani P, Franceschi C, Brigidi P. Ageing and gut microbes: perspectives for health maintenance and longevity. Pharmacol Res. 2013;69(1):11-20.

21. Deschasaux M, Bouter KE, Prodan A, Levin E, Groen AK, Herrema H, Tremaroli V, Bakker GJ, Attaye I, Pinto-Sietsma SJ, et al. Depicting the composition of gut microbiota in a population with varied ethnic origins but shared geography. Nat Med. 2018;24:1526-31.

22. Ghosh TS, Das M, Jeffery IB, O'Toole PW. Adjusting for age improves identification of gut microbiome alterations in multiple diseases. eLife 2020; 9:e50240.

23. Collado MC, Derrien M, Isolauri E, de Vos WM, Salminen S. Intestinal Integrity and Akkermansia muciniphila, a Mucin-Degrading Member of the Intestinal Microbiota Present in Infants, Adults, and the Elderly. Appl Environ Microbiol. 2007;73(23):7767.

24. Shin NR, Lee JC, Lee HY, Kim MS, Whon TW, Lee MS, Bae JW. An increase in the Akkermansia spp. population induced by metformin treatment improves glucose homeostasis in diet-induced obese mice. Gut. 2014;63(5):727-35.

25. Dubourg G, Lagier JC, Armougom F, Robert C, Audoly G, Papazian L, Raoult D. High-level colonisation of the human gut by Verrucomicrobia following broad-spectrum antibiotic treatment. Int J Antimicrob Agents. 2013:41(2):149-55.

26. Guo X, Zhang J, Wu F, Zhang M, Yi M, Peng Y. Different sub-type strains of Akkermansia muciniphila abundantly colonize in southern China. J Appl Microbiol. 2016;120:452-9.

27. Shin NE, Lee JC, Lee HY, et al. An increase in the Akkermansia spp. population induced by metformin treatment improves glucose homeostasis in diet-induced obese mice. Gut. 2014;63(5):727-35.

28. Junjie $Q$, Yingrui L, Zhiming C, Shenghui L, Jianfeng Z, Fan Z, Suisha L, Wenwei Z, Yuanlin G, Dongqian S. A metagenome-wide association study of gut microbiota in type 2 diabetes. Nature. 2012;490(7418):55-60.

\section{Publisher's Note}

Springer Nature remains neutral with regard to jurisdictional claims in published maps and institutional affiliations.
Ready to submit your research? Choose BMC and benefit from:

- fast, convenient online submission

- thorough peer review by experienced researchers in your field

- rapid publication on acceptance

- support for research data, including large and complex data types

- gold Open Access which fosters wider collaboration and increased citations

- maximum visibility for your research: over $100 \mathrm{M}$ website views per year

At BMC, research is always in progress.

Learn more biomedcentral.com/submissions 